\title{
Percutaneous operative treatment of fragility fractures of the pelvis may not increase the general rate of complications compared to non-operative treatment
}

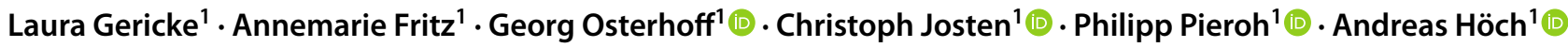

Received: 31 August 2020 / Accepted: 23 March 2021 / Published online: 3 April 2021

(c) The Author(s) 2021

\begin{abstract}
Purpose Despite an increasing number of fragility fractures of the pelvis (FFP) over the last 2 decades, controversy persists on their therapy with special regard to potential complications. Therefore, the present study compared the complication rates and in-hospital mortality of non-operative therapy, percutaneous treatment and open reduction and internal fixation (ORIF) of pelvic fractures in elderly patients.

Methods All consecutive patients treated for FFP between January 2013 and December 2017 aged 65 years or older were retrospectively identified from an institutional database. Demographic data and specific patient data were collected with a special focus on pre-existing comorbidities. General and surgical complications, hospital length of stay (LOS) and mortality rates were compared.

Results 379 patients $(81.3 \pm 7.5$ years; $81 \%$ female) were identified, 211 (55.7\%) were treated non-operatively, 74 (19.5\%) percutaneously and $94(24.8 \%)$ with ORIF. The rate of general complications did not differ between treatment groups (non-operative: $21.8 \%$; percutaneous: $28.4 \%$; ORIF: $33.0 \%$; $p=0.103$ ). Surgery-related complications were twofold more frequent in the ORIF group as than in the percutaneously treated group (18.1\% vs. 9.5\%). The LOS differed significantly (non-operatively: $8.9 \pm 7.1$ days; percutaneous: $16.6 \pm 8.2$ days; ORIF: $19.3 \pm 12.8$ days; $p<0.001$ ). Hospital mortality rate was higher in patients with ORIF (5.3\%) than percutaneous treatment $(0 \%)(p=0.044)$.

Conclusions Complication rates and hospital mortality in elderly patients with FFPs are high and associated with long LOS. For surgical treatment of FFPs, the complication rate and mortality can be significantly reduced using percutaneous procedures compared to ORIF. Therefore, percutaneous surgery should be preferred where possible.
\end{abstract}

Keywords Fragility fractures of the pelvis $\cdot$ Non-operative treatment $\cdot$ Operative treatment $\cdot$ Complications $\cdot$ Mortality

\section{Introduction}

Despite an increasing number of fragility fractures of the pelvis (FFP) over the last 2 decades [1-3], controversy persists on their appropriate treatment with special regard to potential complications. Independent of the treatment, the main goal for these fractures is to reduce pain and thus accelerate patient`s mobilization [4-6]. In several cases

Andreas Höch

Andreas.Hoech@medizin.uni-leipzig.de

1 Department of Orthopedics, Trauma and Plastic Surgery, University Hospital Leipzig, University of Leipzig, Liebigstrasse 20, 04103 Leipzig, Germany non-operative treatment including adequate pain therapy, physical therapy and osteoporosis therapy fulfills these aims [7-9].

In case of unstable fractures or in case of failure of nonoperative treatment, surgical stabilization is recommended [6]. In recent years, various procedures have been developed or adopted from conventional pelvic surgery for young patients [10-13]. Percutaneous procedures are increasingly used in the treatment of FFP in old and frequently comorbid patients. In contrast to young patients, the focus is not necessarily on anatomical reconstruction, but on stabilization and the resulting pain relief and fracture healing [14].

Complication rates in elderly patients with a pelvic fracture can be up to $58 \%$ even with non-operative treatment of supposed simple fractures $[8,15]$. Data on complication rates after operative treatment of FFP are only available in 
restricted numbers and are usually limited to case series of specific surgical techniques $[16,17]$.

Therefore, in this study we compared the complication rates and in-hospital mortality of non-operative therapy and percutaneous surgery and open reduction and internal fixation (ORIF).

\section{Patients and methods}

The present study was approved by the local ethics committee (151/17-ek).

\section{Patients}

All consecutive patients aged 65 years or older treated for FFP were retrospectively identified from an institutional database. According to the inclusion and exclusion criteria, 379 patients were available for evaluation (Fig. 1).

\section{Treatment}

Patients were divided into three groups depending on their treatment: non-operative treatment, percutaneous surgery, and ORIF. Percutaneous surgery was defined as the use of iliosacral/transsacral screws (augmentation was used depending to the surgeon), pubic ramus screws and supraacetabular screws. ORIF included all open approaches (modified Stoppa approach or first window of the ilioinguinal approach) or their combination followed by internal fixation by plates or screws. Spinopelvic fixation was classified as an open method in this study. If only a part of the surgery was performed open, it was considered to belong to the ORIF group.

According to our institutional protocol, FFP I and FFP II were managed non-operatively using pain killers and mobilization under physiotherapeutic supervision. In case mobilization failed within 4-5 days, percutaneous surgical treatment or ORIF was performed. FFP III and FFP IV with

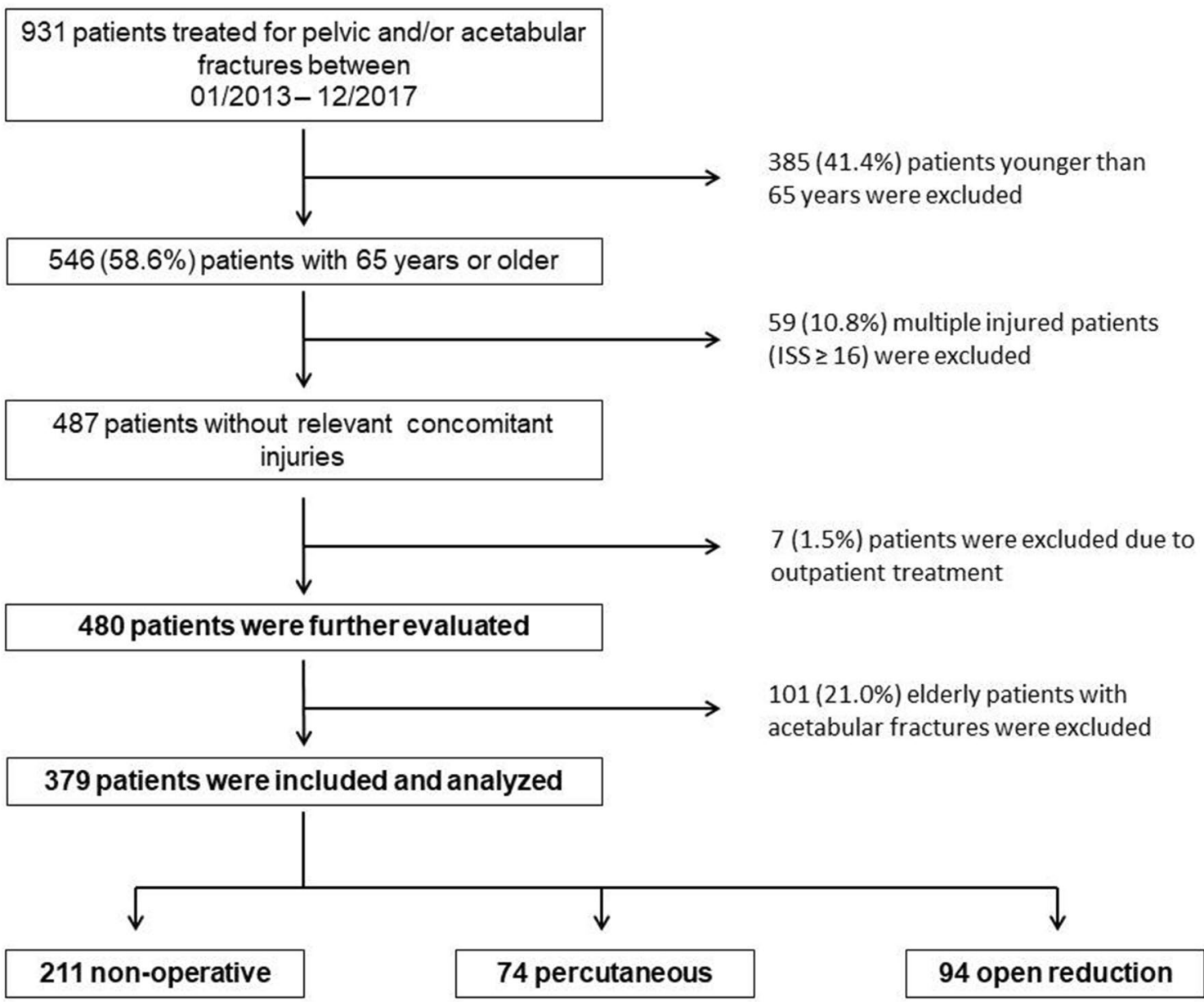

Fig. 1 Study protocol. Patients treated for fragility fractures of the pelvis between 01/2013 and 12/2017 with complete data and aged $\geq 65$ years were evaluated. Patients with multiple injuries and patients treated solely in the outpatient clinic were excluded (ISS $=$ Injury Severity Score) 
corresponding symptoms were indicated for surgical therapy if there were no contraindications or the patient refused surgery. All patients were allowed full weight-bearing postoperatively, regardless of treatment.

\section{Data acquisition}

Patient`s demographics and the following comorbidities were recorded: diabetes mellitus; arterial hypertension; chronic heart failure (CHF); chronic obstructive pulmonary disease (COPD); osteoporosis; renal insufficiency; systemic malignancy; and dementia.

All fractures were classified according to Rommens and Hofmann with FFP classification and retrospectively verified by the senior author [6].

The following documented acute major complications that required specific treatment were evaluated: pneumonia, acute cardiac event, thromboembolism, stroke, multiple organ failure, systemic inflammatory response syndrome (SIRS), sepsis, and delirium. Treated urinary tract infections (UTI) were not counted as major complications, as there are concerns about the documentation and definition of the attending clinician.

Surgical complications documented include the following: unexpected intraoperative bleeding, iatrogenic nerve damage, implant malpositioning, surgical site infections, and implant failure with loss of reduction.

In addition, the time to surgery, length of hospital stay (LOS) and mortality were recorded and evaluated.

\section{Statistical analysis}

Statistical analyses were performed in SPSS 24.0 (SPSS Inc., Chicago, IL, USA). Unless otherwise denoted, data were summarized as mean with standard deviation (SD).

Primary outcome was the occurrence of acute complications during the hospitalization. The hypothesis was that the complication rate as well as LOS and mortality depend on the extent of the surgical therapy. In order to assess possible interfering factors, the baseline characteristics and pre-existing conditions were compared between nonoperative, percutaneous and ORIF groups. For all analyzed data, a normal distribution could be determined using the Shapiro-Wilk test. Nominal data were compared with the Chi-square test and for continuous data the Kruskal-Wallis test and Mann-Whitney $U$ test were used depending on the number of compared groups. The level of significance was defined as $p<0.05$.

\section{Results}

Of 379 patients included (age: $81.3 \pm 7.5$ years; $81 \%$ female), $211(55.7 \%)$ were treated non-operatively, 74 (19.5\%) percutaneously and 94 (24.8\%) with ORIF. Patient's demographics and their comparison in relation to their treatment group are presented in Table 1. A significant difference of age distribution was found between the treatment groups with oldest patient in non-operative group and the youngest in the ORIF group $(p<0.001)$.

Almost all patients had pre-existing comorbidities (97.6\%); significantly different between the groups was the incidence of dementia that correlated with non-operative treatment (Table 2).

The distribution of fractures and the treatment of the respective fracture types are shown in Figs. 2 and 3.

Summarizing the general complications including UTIs and comparing them yielded non-significant differences between treatment groups (non-operative: $21.8 \%$; percutaneous: $28.4 \%$; ORIF: $33.0 \%$; $p=0.103$ ) (Table 3).

Surgery-related complications occurred in a total of $15.7 \%$ of patients and had a twofold higher incidence in the ORIF group compared to the percutaneously treated group $(18.1 \%$ vs. $9.5 \% ; p=0.113)$. Predominant is the difference in the infection rate $(12.8 \%$ vs. $0 \% ; p<0.001)$. In $10 / 12$ patients, the implant was preserved after revision surgery due to infection, two patients received a change from a
Table 1 Baseline data compared between treatment groups

\begin{tabular}{|c|c|c|c|c|c|}
\hline & \multirow{2}{*}{$\begin{array}{l}\text { Total } \\
n=379\end{array}$} & \multicolumn{3}{|l|}{ Treatment } & \multirow[t]{2}{*}{$p$} \\
\hline & & $\begin{array}{l}\text { Non-operative } \\
n=211\end{array}$ & $\begin{array}{l}\text { Percutaneous } \\
n=74\end{array}$ & $\begin{array}{l}\text { ORIF } \\
n=94\end{array}$ & \\
\hline Age $[$ years $($ mean $\pm S D)]$ & $81.3 \pm 7.5$ & $82.8 \pm 7.8$ & $80.4 \pm 6.2$ & $78.7 \pm 6.9$ & $<0.001$ \\
\hline \multicolumn{6}{|l|}{ Gender $[n(\%)]$} \\
\hline Female & $305(80.5)$ & $175(82.9)$ & $60(81.1)$ & $70(74.5)$ & \multirow[t]{2}{*}{$>0.2$} \\
\hline Male & $74(19.5)$ & $36(17.1)$ & 14 (18.9) & $24(25.5)$ & \\
\hline \multicolumn{6}{|l|}{ Comorbidity $[n(\%)]$} \\
\hline None & $9(2.4)$ & $9(4.3)$ & $0(0.0)$ & $0(0.0)$ & \multirow[t]{3}{*}{$<0.01$} \\
\hline$\leq 2$ & $141(37.4)$ & $81(38.4)$ & $21(28.4)$ & $39(41.5)$ & \\
\hline$>2$ & $227(59.9)$ & $121(57.3)$ & $53(71.6)$ & $53(56.4)$ & \\
\hline
\end{tabular}


Table 2 Pre-existing comorbidities compared between treatment groups. Several comorbidities per patient are possible

\begin{tabular}{lccccc}
\hline Comorbidities $[n(\%)]$ & Total & \multicolumn{2}{l}{ Treatment } & \multirow{2}{*}{$p$} \\
\cline { 3 - 4 } & & Non-operative & Percutaneous & ORIF & \\
\hline None & $9(2.4)$ & $9(4.3)$ & $0(0.0)$ & $0(0.0)$ & 0.002 \\
Diabetes mellitus & $92(24.3)$ & $52(24.6)$ & $20(27.0)$ & $20(21.3)$ & 0.722 \\
Arterial hypertension & $315(83.1)$ & $177(83.9)$ & $64(86.5)$ & $74(78.7)$ & 0.550 \\
Osteoporosis & $143(37.7)$ & $71(33.6)$ & $36(48.6)$ & $36(38.3)$ & 0.080 \\
Renal insufficiency & $47(12.4)$ & $22(10.4)$ & $13(17.6)$ & $12(12.8)$ & 0.280 \\
COPD & $52(13.7)$ & $28(13.3)$ & $10(13.5)$ & $14(14.9)$ & 0.906 \\
Chronic heart failure & $86(22.7)$ & $42(19.9)$ & $23(31.1)$ & $21(22.3)$ & 0.155 \\
Systemic malignancy & $35(9.2)$ & $17(8.1)$ & $5(6.8)$ & $13(13.8)$ & 0.176 \\
Dementia & $61(16.1)$ & $46(21.8)$ & $5(6.8)$ & $10(10.6)$ & 0.003 \\
\hline
\end{tabular}

$C O P D$ chronic obstructive pulmonary disease

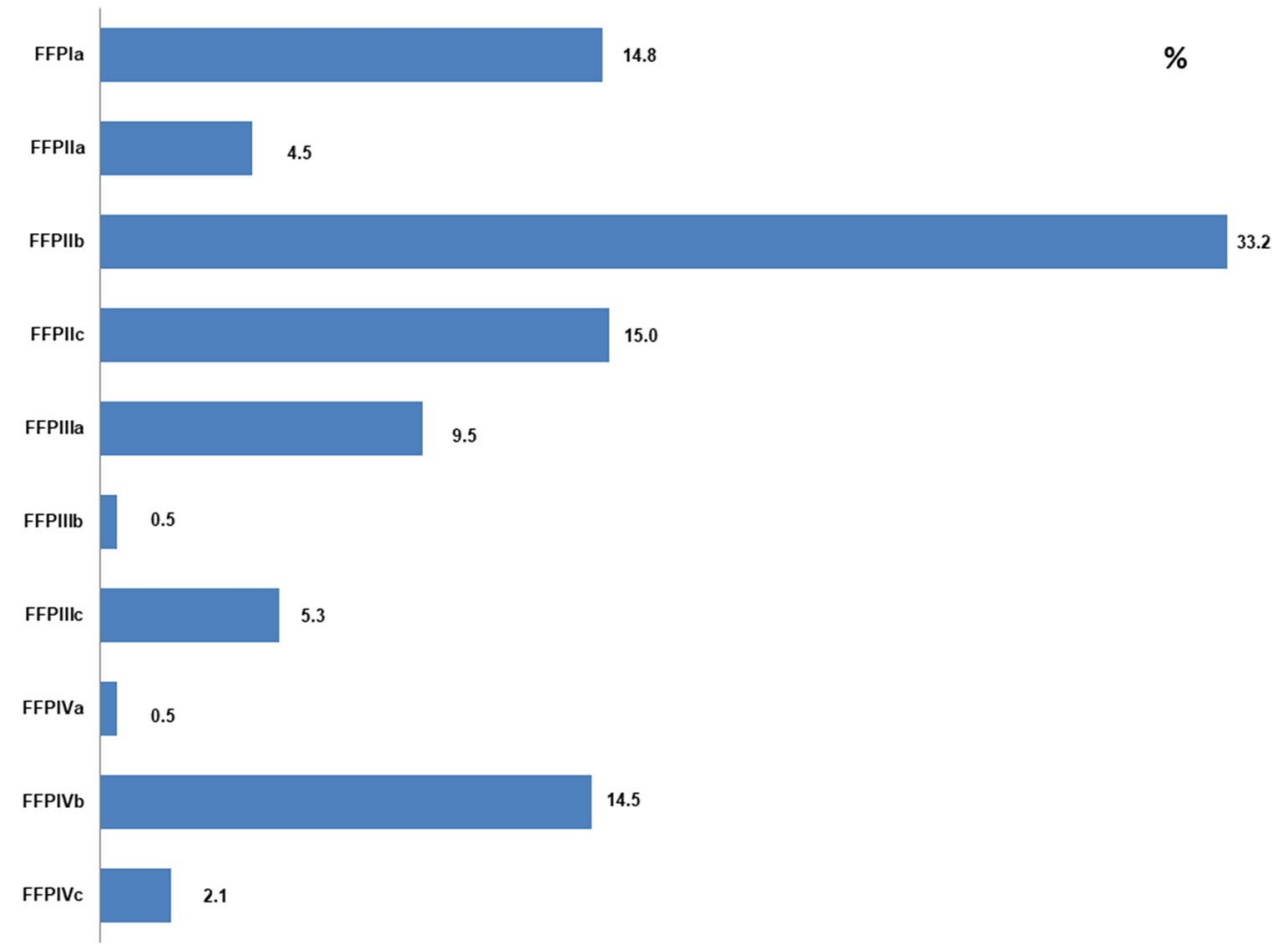

Fig. 2 Fracture distribution of the fragility fractures of the pelvis according to Rommens and Hofmann [6] spinopelvic fixation to sacroiliac screw fixation. Details on the complications are given in Table 4.

The shortest LOS was observed in non-operatively treated patients $(8.9 \pm 7.1$ days $)$, followed by the percutaneously treated $(16.6 \pm 8.2$ days $)$ and the longest remaining were patients with ORIF $(19.3 \pm 12.8$ days $)(p<0.001)$. Preoperative waiting time for percutaneous treated patients was longer than for ORIF $(6.9 \pm 5.0$ days vs. $5.0 \pm 3.8$ days; $p<0.007$ ), but postoperative hospital stay was distinctly shorter for percutaneously treated patients than for ORIF $(9.7 \pm 6.0$ days vs. $14.2 \pm 12.3$ days; $p<0.001)$.

Total hospital mortality rate was $2.9 \%$ and there was no difference between non-operative $(2.8 \%)$ and operative treatment $(3.0 \%)(p=1.0)$ overall. Nevertheless, mortality was significantly higher for ORIF (5.3\%) compared to percutaneous treatment $(0.0 \%)(p=0.044)$.

A detailed data overview depending on the FFP classification and treatment group are presented in the supplemental Table 1.

\section{Discussion}

The aim of this study was to compare the general complication rates of non-operative therapy, percutaneous treatment and ORIF of FFPs in patients aged over 65 years.

Overall, the complication rate and mortality in our investigated patient population is very high and the treatment is 
Fig. 3 Type and frequency of treatment depending on FFP classification

Table 3 General complications. Several complications per patient are possible

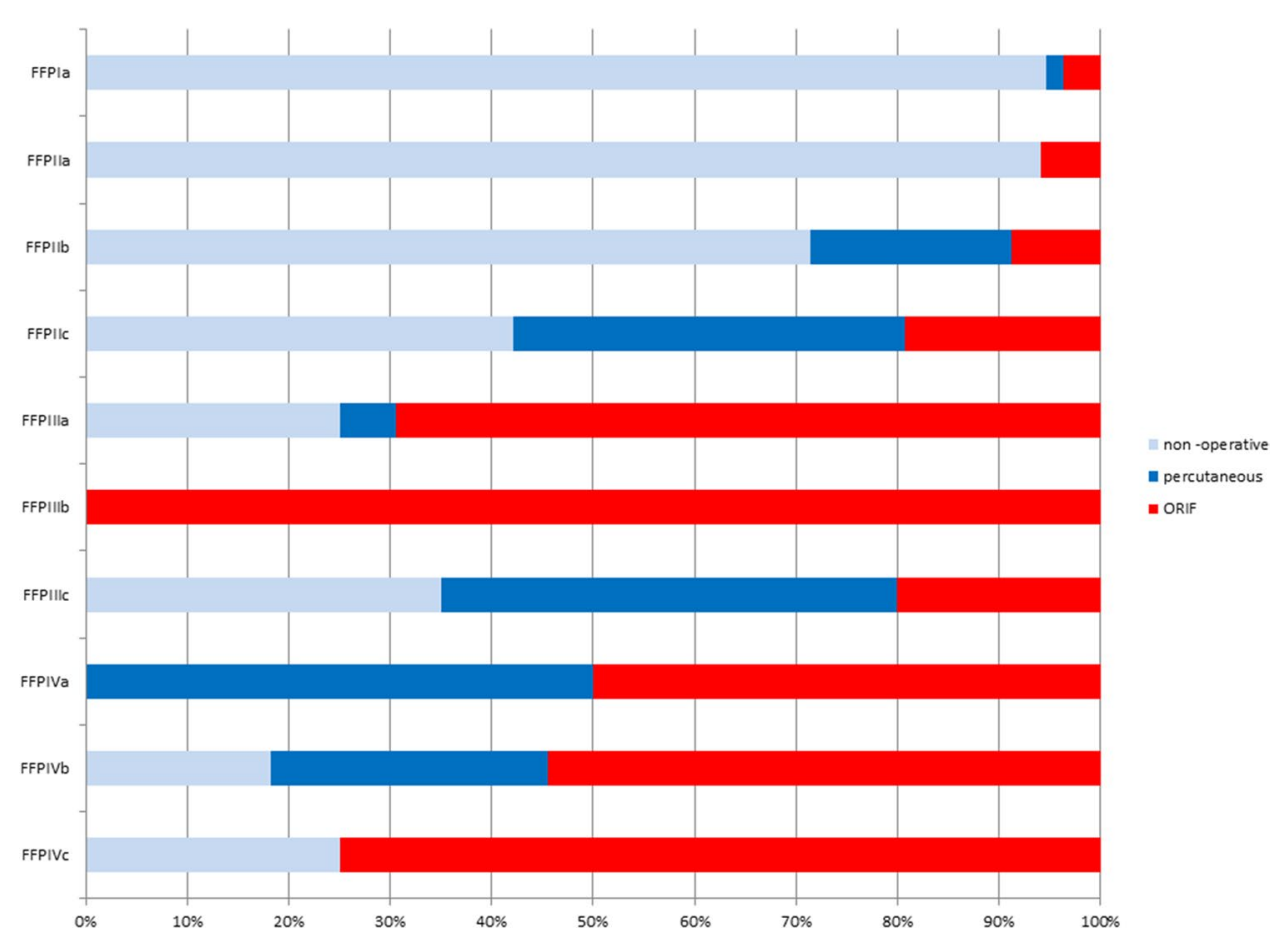

\begin{tabular}{lrllll}
\hline Complications $[n(\%)]$ & Total & \multicolumn{2}{l}{ Treatment } & \multirow{2}{*}{$p$} \\
\cline { 3 - 4 } & & Non-operative & Percutaneous & ORIF & \\
\hline Overall & $36(9.5)$ & $22(10.4)$ & $3(4.1)$ & $11(11.7)$ & 0.193 \\
Multiple organ failure & $9(2.4)$ & $5(2.4)$ & $1(1.4)$ & $3(3.2)$ & 0.739 \\
Pneumonia & $13(3.4)$ & $7(3.3)$ & $2(2.7)$ & $4(4.3)$ & 0.852 \\
Delirium & $9(2.4)$ & $6(2.8)$ & $0(0)$ & $3(3.2)$ & 0.321 \\
Cardiac event & $4(1.1)$ & $2(0.9)$ & $0(0)$ & $2(2.1)$ & 0.397 \\
SIRS/sepsis & $3(0.8)$ & $1(0.5)$ & $0(0)$ & $2(2.1)$ & 0.223 \\
Pulmonary embolism & $1(0.3)$ & $1(0.5)$ & $0(0)$ & $0(0)$ & 0.671 \\
Thrombosis & $1(0.3)$ & $1(0.5)$ & $0(0)$ & $0(0)$ & 0.671 \\
\hline
\end{tabular}

SIRS systemic inflammatory response syndrome
Table 4 Surgery-related complications depending on the technique of surgery

\begin{tabular}{|c|c|c|c|c|}
\hline \multirow{2}{*}{$\begin{array}{l}\text { Complications }[n \\
(\%)]\end{array}$} & \multirow{2}{*}{$\begin{array}{l}\text { Total } \\
n=168\end{array}$} & \multicolumn{2}{|l|}{ Treatment } & \multirow[t]{2}{*}{$p$} \\
\hline & & $\begin{array}{l}\text { percutaneous } \\
n=74\end{array}$ & $\begin{array}{l}\text { ORIF } \\
n=94\end{array}$ & \\
\hline Overall & $24(15.7)$ & $7(9.5)$ & $17(18.1)$ & 0.113 \\
\hline Malpositioning & $5(3.0)$ & $5(6.8)$ & $1(1.1)$ & 0.190 \\
\hline Bleeding & $3(1.8)$ & $1(1.4)$ & $2(2.1)$ & 0.666 \\
\hline Infection & $12(7.1)$ & - & $12(12.8)$ & $<0.001$ \\
\hline Loss of reduction & $2(1.2)$ & $1(1.4)$ & $1(1.1)$ & $>0.05$ \\
\hline Nerve damage & $2(1.2)$ & - & $2(2.1)$ & $>0.05$ \\
\hline
\end{tabular}

Several complications per patient are possible associated with long LOS especially in operative-treated patients. The complication rate increased once again in the patients treated operatively. However, the examination of patients treated percutaneously showed no higher complication rate than in non-operatively treated patients and a significantly lower complication rate than in patients with ORIF.

The general complication rate of $9.5 \%$ was found to coincide with previously published data, which may vary significantly depending on the detection of urinary tract infections $[8,15,18,19]$. For this reason, we analyzed complications without urinary tract infections. Loggers was able to show that, in contrast to purely ventral fractures, the complication 
rate increases significantly from 18 to $44 \%$ when the posterior pelvic ring is involved [20]. Although UTIs were included, this rate was higher than in our study.

Surgical complications were found in $15.7 \%$ of all patients. The most common surgical complication was infection, which was only seen after ORIF in $12.8 \%$ of cases. Ochenjele et al. reported similar rates of complications (overall 15\%; infections 8\%). However, younger and multiple injured patients were included from Ochenjele et al. limiting the comparison [21]. Data on elderly patients with fragility FFP are limited to case series reporting on specific percutaneous surgical techniques [13, 22].

As previously published, we also found a prolonged inpatient stay with a maximum for ORIF of almost 20 days on average $[4,16]$. From the clinical experience, this can be explained partly by the time-consuming perioperative management and the organization of a mostly necessary rehabilitation or nursing care after discharge.

The in-hospital mortality rate of $2.9 \%$ also corresponds to previous reports $(1.3-7.6 \%)$ [16, 23, 24]. This high complication rate in isolated pelvic fractures, which does not usually result from high-energy accidents in old patients, indicate a very fragile patient population with high risk factors [25].

In a recent registry study "general health" was frequently stated as a reason for non-operative treatment and thus influences the surgeons decision [25]. The present study results suggest that dementia leads more often to non-operative treatment. Reasons for this may be an expected higher mortality and complication rate [26, 27]. In addition, the clinical assessment of patients with dementia is significantly more difficult, especially with regard to pain. Apart from this factor, no difference could be found between the therapy groups with regard to comorbidities.

Several limitations to the present study must be stated. Due to the retrospective nature of the study, no statement about the treatment decision is possible. Certainly, there is a bias in this examination, as the decision on therapy also depends on comorbidities in individual cases. It is well known that besides chronological age, biological age and activity level of the patients affect the therapy decisions. To be able to compare this more objectively, a validated frailty index should be used in prospective studies.

Not every patient can be treated non-operatively due to persistent pain and immobilization. As is well known, persistent immobilization increases the risk of other complications such as thrombosis, pneumonia, pressure ulcerations, etc. $[16,28-30]$. The complication rate of percutaneous treatment is not significantly higher compared to non-operative therapy and other studies have already shown significant pain reduction with such techniques [13, 22, 31]. Especially for FFP, percutaneous solutions for surgical treatment of almost all fracture types are possible. In elderly patients, the goal of surgical therapy is not necessarily anatomical reduction, but rather stabilization of the fracture to induce and facilitate a healing process and alleviate the patients' pain $[4,5,14,25]$.

Another weakness of this study is the lack of follow-up after discharge. Especially for FFP, we know that it can be a creeping process and a fracture successfully treated nonoperatively at the beginning can also worsen and require surgical treatment later on $[5,17,32]$.

\section{Conclusion}

Inpatient complication rates in elderly patients with FFP are high and associated with prolonged LOS.

In case of operative treatment of FFP in elderly patients with existing comorbidities, the complication rate and mortality can be significantly reduced using percutaneous procedures instead of ORIF. Therefore, a percutaneous procedure should be preferred whenever possible.

Supplementary Information The online version contains supplementary material available at https://doi.org/10.1007/s00068-021-01660-w.

Authors' contributions Conceived and designed the study: LG, GO, AH. Data acquisition: LG, AF. Analyzed the data: LG, GO, AH. Wrote and draft the paper: LG, PP, AH. Approved the final version of the manuscript: LG, AF, GO, PP, CJ, AH.

Funding Open Access funding enabled and organized by Projekt DEAL.

\section{Declarations}

Conflict of interest All authors disclose any conflict of interest with any financial organizations regarding the material discussed in the manuscript. There are no financial and personal relationships of any author with other people or organizations that could inappropriately influence their work.

Open Access This article is licensed under a Creative Commons Attribution 4.0 International License, which permits use, sharing, adaptation, distribution and reproduction in any medium or format, as long as you give appropriate credit to the original author(s) and the source, provide a link to the Creative Commons licence, and indicate if changes were made. The images or other third party material in this article are included in the article's Creative Commons licence, unless indicated otherwise in a credit line to the material. If material is not included in the article's Creative Commons licence and your intended use is not permitted by statutory regulation or exceeds the permitted use, you will need to obtain permission directly from the copyright holder. To view a copy of this licence, visit http://creativecommons.org/licenses/by/4.0/. 


\section{References}

1. Buller LT, Best MJ, Quinnan SM. A nationwide analysis of pelvic ring fractures: incidence and trends in treatment, length of stay, and mortality. Geriatr Orthop Surg Rehabil. 2016;7:9-17. https:// doi.org/10.1177/2151458515616250.

2. Andrich S, Haastert B, Neuhaus E, et al. Epidemiology of pelvic fractures in germany: considerably high incidence rates among older people. PLoS ONE. 2015;10:e0139078. https://doi.org/10. 1371/journal.pone.0139078.

3. Clement ND, Court-Brown CM. Elderly pelvic fractures: the incidence is increasing and patient demographics can be used to predict the outcome. Eur J Orthop Surg Traumatol. 2014;24:1431-7. https://doi.org/10.1007/s00590-014-1439-7.

4. Oberkircher L, Ruchholtz S, Rommens PM, et al. Osteoporotic pelvic fractures. Dtsch Arztebl Int. 2018;115:70-80. https://doi. org/10.3238/arztebl.2018.0070.

5. Rommens PM, Wagner D, Hofmann A. Fragility fractures of the pelvis. JBJS Rev. 2017. https://doi.org/10.2106/JBJS.RVW.16. 00057.

6. Rommens PM, Hofmann A. Comprehensive classification of fragility fractures of the pelvic ring: recommendations for surgical treatment. Injury. 2013;44:1733-44. https://doi.org/10.1016/j. injury.2013.06.023.

7. Kanakaris NK, Greven T, West RM, et al. Implementation of a standardized protocol to manage elderly patients with low energy pelvic fractures: can service improvement be expected? Int Orthop. 2017;41:1813-24. https://doi.org/10.1007/ s00264-017-3567-2.

8. Maier GS, Kolbow K, Lazovic D, et al. Risk factors for pelvic insufficiency fractures and outcome after conservative therapy. Arch Gerontol Geriatr. 2016;67:80-5. https://doi.org/10.1016/j. archger.2016.06.020.

9. Rollmann MF, Herath SC, Holstein JH, et al. Surgical treatment of pelvic ring fractures in the elderly now and then: a pelvic registry study. Aging Clin Exp Res. 2017;29:639-46. https://doi.org/10. 1007/s40520-016-0612-8.

10. Schmitz P, Baumann F, Grechenig S, et al. The cement-augmented transiliacal internal fixator (caTIFI): an innovative surgical technique for stabilization of fragility fractures of the pelvis. Injury. 2015;46(Suppl 4):S114-20. https://doi.org/10.1016/S00201383(15)30029-2.

11. König A, Oberkircher L, Beeres FJP, et al. Cement augmentation of sacroiliac screws in fragility fractures of the pelvic ring-A synopsis and systematic review of the current literature. Injury. 2019;50:1411-7. https://doi.org/10.1016/j.injury.2019.06.025.

12. Kim W-Y, Lee S-W, Kim K-W, et al. Minimally invasive surgical treatment using "iliac pillar" screw for isolated iliac wing fractures in geriatric patients: a new challenge. Eur J Trauma Emerg Surg. 2019;45:213-9. https://doi.org/10.1007/s00068-018-1046-0.

13. Höch A, Pieroh P, Henkelmann R, et al. In-screw polymethylmethacrylate-augmented sacroiliac screw for the treatment of fragility fractures of the pelvis: a prospective, observational study with 1-year follow-up. BMC Surg. 2017;17:132. https://doi.org/10. 1186/s12893-017-0330-y.

14. Rommens PM. Paradigm shift in geriatric fracture treatment. Eur J Trauma Emerg Surg. 2019;45:181-9. https://doi.org/10.1007/ s00068-019-01080-x.

15. Breuil V, Roux CH, Testa J, et al. Outcome of osteoporotic pelvic fractures: an underestimated severity. Survey of 60 cases. Jt Bone Spine. 2008;75:585-8. https://doi.org/10.1016/j.jbspin.2008.01. 024.
16. Noser J, Dietrich M, Tiziani S, et al. Mid-term follow-up after surgical treatment of fragility fractures of the pelvis. Injury. 2018;49:2032-5. https://doi.org/10.1016/j.injury.2018.09.017.

17. Höch A, Özkurtul O, Pieroh P, et al. Outcome and 2-year survival rate in elderly patients with lateral compression fractures of the pelvis. Geriatr Orthop Surg Rehabil. 2017;8:3-9. https://doi.org/ 10.1177/2151458516681142.

18. Mears SC, Berry DJ. Outcomes of displaced and nondisplaced pelvic and sacral fractures in elderly adults. J Am Geriatr Soc. 2011;59:1309-12. https://doi.org/10.1111/j.1532-5415.2011. 03455.x.

19. van Dijk WA, Poeze M, van Helden SH, et al. Ten-year mortality among hospitalised patients with fractures of the pubic rami. Injury. 2010;41:411-4. https://doi.org/10.1016/j.injury.2009.12. 014.

20. Loggers SAI, Joosse P, Jan Ponsen K. Outcome of pubic rami fractures with or without concomitant involvement of the posterior ring in elderly patients. Eur J Trauma Emerg Surg. 2018. https:// doi.org/10.1007/s00068-018-0971-2.

21. Ochenjele G, Reid KR, Castillo RC, et al. Predictors of unplanned reoperation after operative treatment of pelvic ring injuries. $\mathrm{J}$ Orthop Trauma. 2018;32:e245-50. https://doi.org/10.1097/BOT. 0000000000001170.

22. Eckardt H, Egger A, Hasler RM, et al. Good functional outcome in patients suffering fragility fractures of the pelvis treated with percutaneous screw stabilisation: assessment of complications and factors influencing failure. Injury. 2017;48:2717-23. https://doi. org/10.1016/j.injury.2017.11.002.

23. Taillandier J, Langue F, Alemanni M, et al. Mortality and functional outcomes of pelvic insufficiency fractures in older patients. Joint Bone Spine. 2003;70:287-9.

24. Morris RO, Sonibare A, Green DJ, et al. Closed pelvic fractures: characteristics and outcomes in older patients admitted to medical and geriatric wards. Postgrad Med J. 2000;76:646-50. https://doi. org/10.1136/pmj.76.900.646.

25. Höch A, Pieroh P, Gras F, et al. Age and "general health"-beside fracture classification-affect the therapeutic decision for geriatric pelvic ring fractures: a German pelvic injury register study. Int Orthop. 2019. https://doi.org/10.1007/s00264-019-04326-w.

26. Hu F, Jiang C, Shen J, et al. Preoperative predictors for mortality following hip fracture surgery: a systematic review and metaanalysis. Injury. 2012;43:676-85. https://doi.org/10.1016/j.injury. 2011.05.017.

27. Morrison RS, Siu AL. Survival in end-stage dementia following acute illness. JAMA. 2000;284:47-52. https://doi.org/10.1001/ jama.284.1.47.

28. Fritz A, Gericke L, Höch A, et al. Time-to-treatment is a risk factor for the development of pressure ulcers in elderly patients with fractures of the pelvis and acetabulum. Injury. 2020;51:352-6. https://doi.org/10.1016/j.injury.2019.12.007.

29. Teasell R, Dittmer DK. Complications of immobilization and bed rest. Part 2: Other complications. Can Fam Physician. 1993;39:1440-2.

30. Dittmer DK, Teasell R. Complications of immobilization and bed rest. Part 1: musculoskeletal and cardiovascular complications. Can Fam Physician. 1993;39:1428-32.

31. Caviglia H, Mejail A, Landro ME, et al. Percutaneous fixation of acetabular fractures. EFORT Open Rev. 2018;3:326-34. https:// doi.org/10.1302/2058-5241.3.170054.

32. Rommens PM, Arand C, Hopf JC, et al. Progress of instability in fragility fractures of the pelvis: an observational study. Injury. 2019. https://doi.org/10.1016/j.injury.2019.08.038. 